\title{
Mitochondrial Neurogastrointestinal Encephalomyopathy (MNGIE):
}

\section{Position Paper on Diagnosis, Prognosis and Treatment by the MNGIE}

\section{International Network}

\section{Running Title: Position Paper on MNGIE}

Michio Hirano ${ }^{1}$, Valerio Carelli ${ }^{2,3}$, Roberto De Giorgio ${ }^{4}$, Loris Pironi ${ }^{5}$, Anna Accarino ${ }^{6}$, Giovanna Cenacchi ${ }^{2}$, Roberto D’Alessandro ${ }^{3}$, Massimiliano Filosto ${ }^{7}$, Ramon Marti $^{8}$, Francesco Nonino ${ }^{3}$, Antonio Daniele Pinna ${ }^{9}$, Elisa Baldin ${ }^{3}$, Bridget Elizabeth Bax ${ }^{10}$, Alessio Bolletta $^{11}$, Riccardo Bolletta ${ }^{11}$, Elisa Boschetti ${ }^{12}$, Matteo Cescon ${ }^{13}$, Roberto D’Angelo ${ }^{14}$, Maria Teresa Dotti ${ }^{15}$, Carla Giordano ${ }^{16}$, Laura Ludovica Gramegna ${ }^{2,3}$, Michelle Levene $^{10}$, Raffaele Lodi ${ }^{2,3}$, Hanna Mandel ${ }^{17}$, Maria Cristina Morelli ${ }^{18}$, Olimpia Musumeci ${ }^{19}$, Alessia Pugliese $^{19}$, Mauro Scarpelli ${ }^{20}$, Antonio Siniscalchi ${ }^{21}$, Antonella Spinazzola ${ }^{22}$, Galit Tal ${ }^{23}$, Javier Torres-Torronteras ${ }^{8}$, Luca Vignatelli ${ }^{3}$, Irina Zaidman ${ }^{24}$, Heinz Zoller ${ }^{25}$, Rita Rinaldi ${ }^{14}$, Massimo Zeviani ${ }^{26}$

\section{Affiliations:}

${ }^{1}$ Department of Neurology, Columbia University Irving Medical Center, New York, New York, USA

${ }^{2}$ Department of Biomedical and Neuromotor Sciences, University of Bologna, Italy

${ }^{3}$ IRCCS Istituto delle Scienze Neurologiche di Bologna, Bologna, Italy

${ }^{4}$ Department of Morphology, Surgery and Experimental Medicine, University of Ferrara, Ferrara, Italy

This article has been accepted for publication and undergone full peer review but has not been through the copyediting, typesetting, pagination and proofreading process which may lead to differences between this version and the Version of Record. Please cite this article as doi: $10.1002 /$ jimd. 12300 
${ }^{5}$ Clinical Nutrition and Metabolism Unit and Center for Chronic Intestinal Failure, Department of Digestive System, St. Orsola-Malpighi Hospital, University of Bologna, Bologna, Italy

${ }^{6}$ Digestive System Research Unit, University Hospital Vall d'Hebron; Centro de Investigación Biomédica en Red de Enfermeda des Hepáticas y Digestivas (CIBEREHD); Departament de Medicina, Universitat Autònoma de Barcelona, Barcelona, Spain ${ }^{7}$ Center for Neuromuscular Diseases, Unit of Neurology, Azienda Socio Sanitaria Territoriale degli Spedali Civili and University of Brescia, Brescia, Italy

${ }^{8}$ Vall d’Hebron Research Institute, Autonomous University of Barcelona; Centro de Investigación Biomédica en Red de Enfermedades Raras (CIBERER), Barcelona, Spain ${ }^{9}$ University of Bologna, St. Orsola-Malpighi Hospital, Bologna, Italy

${ }^{10}$ Institute of Molecular and Clinical Sciences, St George’s University of London, London, United Kingdom

${ }^{11}$ Mitochondrial Patients, Mitocon ONLUS, Rome, Italy

${ }^{12}$ Department of Medical and Surgical Sciences, University of Bologna, Bologna, Italy

${ }^{13}$ General Surgery and Transplant Unit, Department of Medical and Surgical Sciences, University of Bologna, Bologna, Italy

${ }^{14}$ IRCCS Istituto delle Scienze Neurologiche di Bologna, UOC Interaziendale Metropolitana (NeuroMet), - Neurologia AOU S.Orsola-Malpighi, Bologna, Italy

${ }^{15}$ Neurological and Metabolic Diseases Clinic, Siena Hospital, Department of Medicine, Surgery and Neuroscience, University of Siena, Siena, Italy 
${ }^{16}$ Department of Radiological, Oncological and Pathological Sciences, Sapienza, University of Rome, Umberto I Policlinic, Rome, Italy

${ }^{17}$ Institute of Human Genetics and Inherited Metabolic Disorders, Galilee Medical Center, Nahariya, Israel

${ }^{18}$ Department for Care of Organ Failures and Transplants, Internal Medicine for the Treatment of Severe Organ Failures, St. Orsola-Malpighi Hospital, Bologna, Italy ${ }^{19}$ Department of Clinical and Experimental Medicine, University of Messina, Messina, Italy ${ }^{20}$ Neurology Unit, Department of Neuroscience, Azienda Ospedaliero Universitaria Integrata Verona, Verona, Italy

${ }^{21}$ Anaesthesiology Intensive Care and Transplantation Unit, St. Orsola-Malpighi Hospital, Bologna, Italy

${ }^{22}$ Department of Clinical Movement Neurosciences, Royal Free Campus, University College of London, Queen Square Institute of Neurology, London, United Kingdom

${ }^{23}$ Metabolic Unit, Ruth Rappaport Children's Hospital, Rambam Health Care Campus, Haifa, Israel

${ }^{24}$ Department of Bone Marrow Transplantation, Hadassah University Medical Center, Jerusalem, Israel

${ }^{25}$ Department of Internal Medicine I, Medical University of Innsbruck, Innsbruck, Austria ${ }^{26}$ Department of Neurosciences, Veneto Institute of Molecular Medicine, University of Padova, Padova, Italy 
Number of Tables: $n=3$ and Number of Figures: $n=2$

Colour Figures: none

\section{Corresponding Author:}

Rita Rinaldi, M.D.

IRCCS Istituto delle Scienze Neurologiche di Bologna, UOC Interaziendale Metropolitana (NeuroMet), - Neurologia AOU S.Orsola-Malpighi, Via Albertoni 15, 40138, Bologna, Italy. Phone: +390512142641 or +390512142652

Fax: +390512142640

E-mail: rita.rinaldi@aosp.bo.it 


\section{SUMMARY}

Mitochondrial Neurogastrointestinal Encephalomyopathy (MNGIE) is a rare autosomal recessive disease caused by TYMP mutations and thymidine phosphorylase (TP) deficiency. Thymidine and deoxyuridine accumulate impairing the mitochondrial DNA maintenance and integrity. Clinically, patients show severe and progressive gastrointestinal and neurological manifestations. The onset typically occurs in the second decade of life and mean age at death is 37 years. Signs and symptoms of MNGIE are heterogeneous and confirmatory diagnostic tests are not routinely performed by most laboratories, accounting for common misdiagnosis. Factors predictive of progression and appropriate tests for monitoring are still undefined. Several treatment options showed promising results in restoring the biochemical imbalance of MNGIE. The lack of controlled studies with appropriate follow-up accounts for the limited evidence informing diagnostic and therapeutic choices. The International Consensus Conference (ICC) on MNGIE, held in Bologna, Italy, on March $30^{\text {th }}-31^{\text {st }}, 2019$, aimed at an evidence-based consensus on diagnosis, prognosis and treatment of MNGIE among experts, patients, caregivers and other stakeholders involved in caring the condition. The conference was conducted according to the National Institute of Health Consensus Conference methodology. A consensus development panel formulated a set of statements and proposed a research agenda. Specifically, the ICC produced recommendations on: (1) diagnostic pathway; (2) prognosis and the main predictors of disease progression; (3) efficacy and safety of treatments; and (4) research priorities on diagnosis, prognosis and treatment. The Bologna ICC on diagnosis, management and treatment of MNGIE provided evidence-based guidance for clinicians incorporating patients' values and preferences. 


\section{SYNOPSIS}

This is the first International Consensus Conference (ICC) aimed at an evidence-based consensus on diagnosis, prognosis and treatment among experts, patients, caregivers and other stakeholders involved in MNGIE. The ICC provided recommendations on diagnostic pathway, prognosis and the main predictors of disease progression, efficacy and safety of treatments, and, finally, identified priorities on cogent research topics on MNGIE.

\section{ABBREVIATION LIST}

CAPD: continuous ambulatory peritoneal dialysis; CDP: consensus development panel; CPEO: chronic progressive external ophthalmoplegia; dThd: thymidine; dUrd: deoxyuridine; EE-TP: erythrocyte encapsulated TP; EWGs: expert workgroups; GI: gastrointestinal; HD: hemodialysis; HSCT: hematopoietic stem cell transplantation; ICC: International Consensus Conference; MNGIE: Mitochondrial Neurogastrointestinal Encephalomyopathy; mtDNA: mitochondrial DNA; OLT: orthotopic liver transplantation; PEG: percutaneous endoscopic gastrostomy; QoL: quality of life SC: scientific committee; SIBO: small intestinal bacterial overgrowth; TC: technical committee; TP: thymidine phosphorylase.

ACKNOWLEDGEMENTSThe authors thank Rosanna Foderà for her translation support to patients with MNGIE during the conference and Giulia Amore, Umberto Pensato and Vincenzo Mastrangelo for their help in the organization of the conference. 


\section{COMPLIANCE WITH ETHICS GUIDELINES}

\section{CONFLICT OF INTEREST}

Valerio Carelli is a consultant for Santhera Pharmaceuticals, GenSight and Stealth

BioTherapeutics and has received research support from Santhera Pharmaceuticals and Stealth BioTherapeutics.

Bridget Elizabeth Bax has received a travel grant and licence fee payment from Orphan technologies and honorariums from Recordati Rare Diseases Fondation d'entreprise and the European Science Foundation.

Michio Hirano received research support from Entrada Therapeutics.

Giovanna Cenacchi, Rita Rinaldi, Heinz Zoller, Francesco Nonino, Luca Vignatelli, Roberto D’Alessandro, Massimiliano Filosto, Maria Teresa Dotti, Hanna Mandel, Laura Ludovica Gramegna, Olimpia Musumeci, Matteo Cescon, Roberto D’Angelo, Alessia Pugliese, Antonella Spinazzola, Elisa Boschetti, Javier Torres-Torronteras, Irina Zaidman, Antonio Siniscalchi, Roberto De Giorgio, Maria Cristina Morelli, Carla Giordano, Elisa Baldin, Loris Pironi, Ramon Martí, Galit Tal, Michelle Levene, Anna Accarino, Raffaele Lodi, Alessio Bolletta, Riccardo Bolletta, Massimo Zeviani, Antonio Daniele Pinna and Mauro Scarpelli declare that they have no conflict of interest. 


\section{AUTHORS’ CONTRIBUTION}

V.C., R.D.G, L.P., R.R., E. Ba., F.N, L.V. participated to the planning, conducting and reporting of the project; R.B, A.B M.H, R.M., A.P., R.DA., E. Bo. participated to the planning and conduction of the project.

B.E.B. contributed to the conduction and reporting of the project.

L.L.G., M.S., A.S., G.T., C.G., O.M., M.C.M., M. C., M.T.D., M. L., H.M., A. S., J. T-T, I.

Z., H.Z. contributed to the conduction of the project.

\section{ETHICS}

This article does not contain any studies with human or animal subjects performed by any of the authors. No ethical approval was required.

\section{STUDY FUNDING}

Azienda Ospedaliero-Universitaria di Bologna Policlinico St. Orsola-Malpighi and IRCCS, Istituto delle Scienze Neurologiche di Bologna (Bologna, Italy).

\section{SEARCH TERMS}

Mitochondrial Neurogastrointestinal Encephalomyopathy; MNGIE; enzyme replacement; mitochondrial disease; TYMP; thymidine phosphorylase, consensus conference. 


\section{INTRODUCTION}

Mitochondrial Neurogastrointestinal Encephalomyopathy (MNGIE) is an autosomal recessive disease caused by mutations in the thymidine phosphorylase gene (TYMP)(Pacitti et al 2018). TYMP encodes for thymidine phosphorylase (TP), which catabolizes thymidine (dThd) and deoxyuridine (dUrd) into their respective bases. TYMP mutations markedly reduce/abolish TP activity leading to accumulation of dThd and dUrd and mitochondrial DNA (mtDNA) defects.MNGIE is an ultra-rare condition, characterized by severe gastrointestinal (GI) and neurological symptoms(D'Angelo et al 2016) that is often misdiagnosed. Although the disease is progressive and fatal, natural history is still uncharacterized(Nishino et al 2000; Garone et al 2011; Corazza et al 2019). Various experimental therapeutic approaches aimed to the temporary enzyme replacement, e.g. erythrocyte encapsulated TP (EE-TP) infusions (Pacitti et al 2018), or permanent restoration of TP activity through hematopoietic stem cell transplantation (HSCT) (Halter et al 2015) and orthotopic liver transplantation (OLT) (De Giorgio et al 2016). Since the severity of GI symptoms influences treatment success, timing of HSCT and OLT is crucial (Halter et al 2015; De Giorgio et al 2016). Possible future options include gene therapy, which has shown pre-clinical efficacy (Torres-Torronteras et al 2014; Cabrera-Pérez et al 2015; TorresTorronteras et al 2016; Torres-Torronteras et al 2018; Yadak et al 2018; Cabrera-Pérez et al 2019).

An International Consensus Conference (ICC) was held to produce an unbiased, evidencebased assessment on MNGIE, leading to a consensus and guidance on the following areas: (I) diagnostic pathway; (II) prognosis and main predictors of disease progression; (III) efficacy 
and safety of treatments.

\section{METHOD}

\subsection{Panel/experts selection}

The Bologna MNGIE ICC was organized and promoted by the Azienda OspedalieroUniversitaria di Bologna, Policlinico S.Orsola-Malpighi, and the IRCCS Istituto delle Scienze Neurologiche di Bologna, Italy, according to the NIH Consensus Development Program methodology(Nair et al 2011) and the Methodological Handbook of the Italian National Guideline System(Candiani et al 2009). The members of the technical committee (TC) and scientific committee (SC), the expert workgroups (EWGs) and the consensus development panel (CDP) were invited based on their expertise in the field, ensuring the participation of all the clinical and non-clinical stakeholders (including patients) and a broad involvement of healthcare professionals from all the clinical aspects of MNGIE. Researchers were identified based on a review of the main authors in the field. The official language of the conference was English supported by a professional translator. A declaration of interest form was signed by every participant. Of the 36 stakeholders invited to the ICC, four declined and five accepted but did not participate. One member attended via teleconference.

\subsection{The assignment, scoping and assessment stages}

The assignment, scoping and assessment stages occurred between January 2018 and March 2019. The ICC took place in Bologna on March $30^{\text {th }}$ and $31^{\text {st }}$. During assignment, the SC appointed a TC, a CDP and three EWGs (Appendix 1). The SC identified three MNGIE 
topics (diagnosis, prognosis and treatment) and questions to be addressed by the EWGs (scoping). Assessment of the evidence was carried out by the TC through a systematic literature search with evidence mapping (Bragge et al 2011) according to the PRISMA guidelines (Moher et al 2009) (Appendix 2). Studies of any design, in English language, published in full on peer reviewed journals, reporting original data on diagnosis, prognosis, and/or treatment of MNGIE on humans were searched on MEDLINE and the Cochrane Central Register of Controlled Trials in January 2018 and 2019, and finally updated in May 2020. Retrieved studies were selected independently by LV, EB and RD. Disagreement was resolved by discussion. Each study was graded according to four classes of methodological quality (from Class I, highest quality to Class IV, lowest quality) according to the Classification of Evidence Schemes of the Clinical Practice Guideline Process Manual of the American Academy of Neurology (Appendix 3) (Gronseth et al 2011) and appraised by the EWGs to draft answers to the questions posed by the SC. During the ICC the scientific evidence and the answers to the questions were presented by the EWGs. The final statements by the CDP were presented at the end of day 2 to the audience including stakeholders and general public.

\section{RESULTS}

The literature search retrieved 1,305 citations after duplicate removal; 1,146 were excluded because the covered topic was not of interest for our review (Appendix 2). Of the 159 full text articles selected, 81 were excluded mostly because they were animal studies or studies describing genetic mutations causing the clinical manifestations of MNGIE. The 78 selected 
articles were submitted to the three working groups. Since 36 of them were out of scope regarding the specific topics, 42 were used for the statements ( 4 of them were assigned to two topics each). The CDP issued the following Position Statements on diagnosis, prognosis and treatment of MNGIE. In Appendix 4 a summary of the scientific evidence and rationale for the statements is presented.

\subsection{Position Statements on the Diagnosis of MNGIE}

The following statements are based on 7 Class III level studies (case series with controls) (Spinazzola et al 2002; Nishigaki et al 2003; Marti et al 2004; Valentino et al 2007; Mohamed et al 2014; Gramegna et al 2018; Kipper et al 2020), 7 Class IV level studies (two case series and five case reports) (Millar et al 2004; Marti et al 2005; Giordano et al 2006; Filosto et al 2011; Garone et al 2011; Scarpelli et al 2013; Corazza et al 2019) and expert opinion.

\subsubsection{Clinical elements that can indicate MNGIE}

MNGIE can be suspected when one or more of the following clinical cardinal elements are present:

- Symptoms and signs of otherwise unexplained GI dysmotility

- Thin constitution/cachexia, even with normal food behaviour and nutritional intake

- Neurological features such as ptosis and symptoms suggesting peripheral neuropathy

- A progressive course of the above with frequent misdiagnosis

The features of the full-blown MNGIE typically are: 
- Symptoms onset: childhood, adolescence/young adulthood (typical), adulthood (late onset, $>40$ years)

- GI symptoms/signs: sub-occlusive episodes, nausea, vomiting, early satiety, borborygmi, severe abdominal pain, abdominal distension, dysphagia, constipation and diarrhoea, acute peritonitis due to small bowel perforation

- Unexplained weight loss, thinness, cachexia, even with normal food behaviour and nutritional intake

- Radiological GI signs: small bowel diverticulosis, GI dilation (e.g. gastric or intestinal dilation)

- Neurological symptoms/signs: chronic progressive external ophthalmoplegia (CPEO), ptosis, peripheral neuropathy, hearing loss

- Neuroradiological signs: leukoencephalopathy without other neuroradiological abnormalities

- Metabolic alterations: liver steatosis evolving in cirrhosis, pancreatitis, early onset diabetes mellitus, increased triglyceride levels, elevated plasma lactate

MNGIE is most frequently misdiagnosed as:

- Anorexia nervosa

- GI diseases: Crohn’s disease, coeliac disease, esophagitis and/or gastritis, irritable bowel syndrome, superior mesenteric artery syndrome, Whipple’s disease, chronic intestinal pseudo-obstruction 
- Neurological diseases: chronic inflammatory demyelinating polyneuropathy, CharcotMarie-Tooth disease, other mitochondrial diseases such as CPEO, Kearns-Sayre syndrome

3.1.2 Recommended diagnostic testsCardinal diagnostic tests:

- Swallowing test, gastric emptying and GI manometry (when possible): altered GI motility and transit

- Brain MRI: leukoencephalopathy without any other neuroradiological abnormalities (almost universally present) (Table 1 and Fig. 2)

- Nerve conduction studies: peripheral neuropathy, predominantly demyelinating Ancillary tests:

- Muscle biopsy: ragged-red and COX deficient fibers, deficiencies of respiratory chain enzyme activities, ultrastructurally abnormal mitochondria, and mtDNA depletion, multiple deletions, and somatic point mutations

- Mucosal GI histology of small bowel (to exclude other conditions), and gut full thickness biopsy (when possible)

\subsubsection{Recommended metabolic and genetic tests}

Mandatory tests to confirm MNGIE diagnosis:

- TYMP sequencing: homozygous or compound heterozygous allelic pathogenic variants, no further testing.

- If one variant of uncertain significance or a wild-type sequence is identified, the following biochemical assessments should be performed: 
o TP activity: severely reduced or virtually absent in the buffy coat (below 8\% of the mean of reference TP values; laboratory cutoffs may differ depending on sample processing and biochemical assay). If TP activity is only partially reduced, then it is mandatory to measure plasma dThd and dUrd levels. The diagnosis is excluded if TP activity is normal.

o dThd and dUrd levels: increased in plasma (assessment of urine is unreliable)

Fig. 1 shows the recommended algorithm in persons with suspected MNGIE.

\subsection{Position Statements on the Prognosis of MNGIE}

The following statements are based on one Class II level study (retrospective cohort) (Garone et al 2011), two Class III level study (retrospective cohorts) (Nishino et al 2000; Corazza et al 2019), 4 Class IV level studies (four case reports) (Ionasescu et al 1984; Carrozzo et al 1998; Marti et al 2005; Massa et al 2009) and expert opinion.

\subsubsection{The natural history of MNGIE}

- Mean age at onset: 17.9 years (5 months - 43 years).

- GI symptoms (57\% at onset; $100 \%$ at diagnosis); onset/diagnosis: diarrhea, abdominal pain, borborygmi, vomiting, pseudo-obstruction (32\%- 65\%), weight loss/cachexia (100\%); evolution: diverticulosis/diverticulitis (67\%), hepatopathy (22\%).

- Neurological symptoms / signs (43\% at onset; $100 \%$ at diagnosis); onset/diagnosis/evolution: ocular signs (ptosis, ophthalmoparesis) (74-100\%), 
polyneuropathy (92-100\%), hearing loss (39-45\%), leucoencephalopathy ( $\pm 100 \%)$; cognitive impairment (20\%).

- Symptoms are cumulative and progressive.

- Mean age at death is reported to range between 35 and 37 years; survival 100\% before 19 years and $<5 \%$ after 50 years.

- Death is mainly due to GI and liver complications (intestinal perforation, intestinal bleeding, liver failure, aspiration pneumonia, complications due to small intestinal bacterial overgrowth (SIBO) or infection related to central venous catheter for parenteral nutrition) and cachexia.

Overall survival is the only outcome reported in the literature. Weight loss is an important feature of MNGIE, but data on its prognostic role are lacking.

\subsubsection{Phenotypes of MNGIE}

MNGIE has two different presentations distinguished by age of onset: "Early Onset” (or “Classic”) and “Late Onset” (Table 2). Severity can vary among family members. Available data do not allow a differentiation of clinical phenotypes based on symptoms at onset (GI or neurological). Neurological manifestations may be subtle and insidious leading to late recognition by both patients and physicians. When GI symptoms are the first manifestation, the diagnosis and consequently the appropriate treatment may be significantly delayed because of misdiagnosis. 
In clinical practice, the presence and/or severity of GI involvement are considered a negative prognostic factor, for both morbidity and mortality. Apparently, there is no correlation between genotype, phenotype and outcome. Residual TP activity of 10-15\% has been associated with moderate increases of nucleosides and "Late Onset” MNGIE compared to the “Classic” form.

\subsubsection{Impact of different phenotypes on the natural history of MNGIE and outcomes}

Whether "Classic" ("Early Onset”) and "Late Onset” phenotypes have different disease progression remain unsettled. All patients with Late Onset phenotype reported in the literature were alive at follow-up ranging between 8 to 24 years. In the "Classic" phenotype, age of onset is not related to life expectancy. At present, overall survival after onset is the only available outcome reported in the literature.

\subsubsection{Events indicating disease progression}

The following events can be considered as important milestones related to progression: GI sub-occlusive episode, decompressive percutaneous endoscopic gastrostomy (PEG), aspiration pneumonia, abdominal surgical procedures, septic episode due to SIBO, need for enteral tube feeding or PEG, onset of intestinal failure (and subsequent need of parenteral nutrition), liver cirrhosis, loss of unaided walking ability. Recommended assessments to monitor MNGIE progression are listed in Table 3.

\subsection{Position Statements on the Treatment of MNGIE}


The following statements are based on 25 Class IV level studies (one retrospective cohort, one case series, 24 case reports) (Hirano et al 2006; la Marca et al 2006; Lara et al 2006; Yavuz et al 2007; Moran et al 2008; Filosto et al 2012; Sicurelli et al 2012; Bax et al 2013; Finkenstedt et al 2013; Hussein 2013; Ariaudo et al 2015; Casarez et al 2015; Halter et al 2015; Peedikayil et al 2015; De Giorgio et al 2016; Sivadasan et al 2016; Baker et al 2017; D'Angelo et al 2017; Roeben et al 2017; Chandra et al 2018; Hanbali et al 2018; Levene et al 2018; Yadak et al 2018; Levene et al 2019; Kripps et al 2020) and expert opinion.

\subsubsection{Treatments effective in temporarily restoring the biochemical imbalance}

"Short term" is defined as a period of time required stabilizing a patient waiting for permanent treatment or as compassionate use.

Overall, hemodialysis (HD), continuous ambulatory peritoneal dialysis (CAPD), EE-TP and platelet infusion have been effective in achieving temporary improvement of the biochemical imbalance in several MNGIE patients. However, there are practical limitations, safety issues and unclear clinical effects associated with these approaches.

Specifically:

- EE-TP seems to be effective on a monthly-based administration in terms of biochemical and clinical improvement (4 patients out of 5); mild immunological reaction against bacterial TP mainly with repeated infusions may occur (2 out of 5 patients). EE-TP is currently under clinical evaluation.

- CAPD seems to be well tolerated with anecdotal biochemical efficacy; peritoneal sclerosis due to repeated procedures may be a safety concern. 
- HD has very short-term biochemical effects as nucleosides return to high levels a few hours after the procedure. Disadvantages include the need of venous access, an intensive procedure schedule (3-4 sessions per week) and the possible occurrence of hypotension, fluid overload or infections.

- Platelet infusion has been reported to achieve some biochemical improvement. Safety issues include allergic and immunological reactions.

We suggest consideration of EE-TP or CAPD in patients waiting for a permanent treatment option, or for compassionate use.

\subsubsection{Effective treatments that permanently restore the biochemical imbalance}

Permanent treatment options are aimed at restoring TP resulting in the long-term clearance of dUrd and dThd. The improved biochemical profile is expected to be associated with clinical stabilization (i.e., halting tissue damage progression) or improvement.

- HSCT is effective in permanently restoring the biochemical imbalance. It requires chemotherapy and immunosuppressive therapy and is associated with a high risk for complications and mortality related to therapy, including graft versus host disease.

- OLT is effective for permanent restoration of the biochemical imbalance and it does not require preoperative conditioning. Patients with severe malnutrition or previous episodes of small bowel perforation, sub-occlusion or sepsis related to SIBO could be at high risk of peri- and post-operative complications and should not be considered for OLT; 
metabolic complications such as chronic kidney insufficiency, diabetes or cardiovascular disease related to long-life immunosuppressive therapy may be long-term issues.

\subsubsection{Treatments that improve the patient's health in terms of quality of life (QoL) and}

\section{functional status}

Any temporary or permanent treatment should be considered as soon as diagnosis is confirmed. EE-TP and CAPD are effective with some temporary improvement of QoL and minimal complications. HSCT is effective in the long term for improving QoL and functional status, although limited by a high post-treatment mortality rate (63\%) in severely symptomatic adult patients. OLT is effective in the long term for improving QoL and functional status, although limited evidence is available.

\subsubsection{Appropriateness of treatments that temporarily or permanently restore the}

\section{biochemical imbalance}

Temporary treatments should be considered at any age based on clinical condition, before permanent treatments, e.g. while waiting /on list for either HSCT or OLT. Once the diagnosis

is confirmed, permanent treatments are recommended as early as possible for both HSCT and OLT according to eligibility criteria (e.g. severity). Ideal candidates should be those at an early stage of MNGIE. In patients who are oligosymptomatic, either HSCT or OLT can be considered. HSCT should be considered in pediatric patients and young adults with normal liver function, mild or no GI manifestations (e.g. absence of intestinal pseudo-obstruction, peritonitis, pancreatitis) and in case of matched donors with normal genotype. A busulphan- 
based myeloablative regimen as a preparation to HSCT is recommended. OLT would be the preferred permanent treatment option for patients with progressive liver involvement (i.e. fibrosis and/or abnormal liver function). Transplant from a living donor can be considered only if the donor's TYMP genotype is normal. In fully informed patients who are severely affected by MNGIE and unlikely to survive permanent correction procedures, temporary metabolite restorative treatments (EE-TP and CAPD) should be offered and discussed.

\subsubsection{Assessments predicting the effect of treatment}

The following assessments may be predictive of effects of treatment:

- Serial plasma levels of dThd and dUrd

- Serial TP activity measurement in buffy coat (only for HSCT)

The clinical outcome after treatment depends upon the patient's disease status prior to treatment. Assessments evaluating the disease status at the time of diagnosis may indicate whether a proposed treatment is likely to be effective. The extent of hepatic, GI involvement and cachexia are key indicators of survival, therefore assessments evaluating these aspects may be predictive of treatment outcome. Several clinical outcome assessments may be used to monitor the effect of treatment. Some of these assessments were discussed in the “Prognosis” section.

\section{DISCUSSION}

This is the first consensus statement on MNGIE, prompted by the severity of a condition that, although very rare, affects mainly young adults causing substantial reduction of life 
expectancy and QoL. Several potentially useful treatments can be offered to patients, and more may be soon available. It is important to coordinate the work of clinicians and researchers in order to generate new useful evidence and provide patients with reliable and consistent information about their condition.

\subsection{Main findings}

The ICC developed over two days. During the first day one representative of each of the three EWG presented (in a meeting open to the general audience) the results of a systematic search of the literature relative to the topic. Each representative summarized the scientific evidence and proposed conclusions. Presentations were followed by discussion, moderated by the chair of the Jury and by a methodologist, during which disagreements were resolved and tentative recommendations were drafted. No disagreement required formal voting. Multi-stakeholder involvement in the public discussion was ensured by involving patients’ advocacy organizations.

\subsection{Strengths and limitations}

MNGIE is genetically determined and gene therapies are still at a pre-clinical stage. Nevertheless, promising treatments that could potentially modify the course of the condition are emerging. The efficacy of available treatments is still not completely defined, and probably influenced by the stage of the disease. Due to the rarity of the condition, the evidence on MNGIE is limited to few case reports and small case series. The ICC aimed at reaching for the first time an evidence-based consensus involving researchers, patients and 
their families and healthcare providers on the clinical and instrumental hallmarks of MNGIE, its expected course and the main criteria guiding the choice of the most appropriate treatment in individual patients. Research priorities were also identified (Appendix 5) along with the newborn promoting collaboration and networks.

Our process had several limitations. First, since the available evidence is scanty and of low quality, the provided guidance is mainly based on the opinion of experienced clinicians and researchers, and therefore subject to bias. Adopting a rigorous and explicit methodology and warranting the possibility of discussion in every stage of the process through public presentations compensated this limitation. In order to avoid a prevailing view by medical experts, we ensured a formal participation of a leading patient advocacy association among the stakeholders in the jury that formulated the guidance and prompted questions and comments by patients and their families in the audience during discussion. Personal interests, that could bias the point of view of individuals, were declared by each participant. Secondly, there was a partial overlapping in the composition of the scientific workgroups and the SC, since some of the members of the formers were also part of the latter. This could have been a potential source of bias that we mitigated by creating groups as large as possible, facilitating a plurality of views within different areas. Due to the rarity of MNGIE and its recent discovery, the number of knowledgeable researchers and clinicians was low. Although the conference was international and almost all invited persons accepted the invitation, the total number of participants did not allow a complete separation of roles. 


\section{REFERENCES}

Ariaudo C, Daidola G, Ferrero B, et al (2015) Mitochondrial neurogastrointestinal encephalomyopathy treated with peritoneal dialysis and bone marrow transplantation. Journal of nephrology 28: 125-127.

Baker MK, Schutte CM, Ranchhod N, Brittain D, van Rensburg JE (2017) Transient clinical improvement of a mitochondrial neurogastrointestinal encephalomyopathy-like syndrome after allogeneic haematopoietic stem cell transplantation. BMJ case reports 2017.

Bax BE, Bain MD, Scarpelli M, Filosto M, Tonin P, Moran N (2013) Clinical and biochemical improvements in a patient with MNGIE following enzyme replacement. Neurology 81: 12691271.

Bragge P, Clavisi O, Turner T, Tavender E, Collie A, Gruen RL (2011) The Global Evidence Mapping Initiative: scoping research in broad topic areas. BMC medical research methodology 11: 92.

Cabrera-Pérez R, Torres-Torronteras J, Vila-Julià F, et al (2015) Prospective therapeutic approaches in mitochondrial neurogastrointestinal encephalomyopathy (MNGIE). Expert Opinion on Orphan Drugs 3: 1167-1182.

Cabrera-Pérez R, Vila-Julià F, Hirano M, Mingozzi F, Torres-Torronteras J, Marti R (2019) Alpha-1Antitrypsin Promoter Improves the Efficacy of an Adeno-Associated Virus Vector for the Treatment of Mitochondrial Neurogastrointestinal Encephalomyopathy. Human gene therapy 30: 985-998.

Candiani G, Colombo C, Daghini R, et al (2009) Sistema Nazionale Linee Guida. Manuale Metodologico. Come realizzare una confrenza di consenso. , Milan: Zadig publishing.

Carrozzo R, Hirano M, Fromenty B, et al (1998) Multiple mtDNA deletions features in autosomal dominant and recessive diseases suggest distinct pathogeneses. Neurology 50: 99-106.

Casarez VQ, Zavala AM, Owusu-Agyemang P, Hagan K (2015) Anesthetic Management of a Child with Mitochondrial Neurogastrointestinal Encephalopathy. Case reports in anesthesiology 2015: 453714.

Chandra VS, Lakshmi BS, Padmavathi Devi SVV, et al (2018) Mitochondrial Neurogastrointestinal Encephalomyopathy: A Nonrenal Indication for Peritoneal Dialysis. Indian J Nephrol 28: 310-313.

Corazza G, Pagan C, Hardy G, Besson G, Lombes A (2019) MyoNeuroGastroIntestinal Encephalopathy: Natural History and Means for Early Diagnosis. Gastroenterology 156: 1525-1527.e1524.

D'Angelo R, Rinaldi R, Carelli V, et al (2016) ITA-MNGIE: an Italian regional and national survey for mitochondrial neuro-gastro-intestinal encephalomyopathy. Neurological sciences : official journal of the Italian Neurological Society and of the Italian Society of Clinical Neurophysiology 37: 1149-1151.

D'Angelo R, Rinaldi R, Pironi L, et al (2017) Liver transplant reverses biochemical imbalance in mitochondrial neurogastrointestinal encephalomyopathy. Mitochondrion 34: 101-102.

De Giorgio R, Pironi L, Rinaldi R, et al (2016) Liver transplantation for mitochondrial neurogastrointestinal encephalomyopathy. Annals of neurology 80: 448-455.

Filosto M, Scarpelli M, Tonin P, et al (2012) Course and management of allogeneic stem cell transplantation in patients with mitochondrial neurogastrointestinal encephalomyopathy. Journal of neurology 259: 2699-2706.

Filosto M, Scarpelli M, Tonin P, et al (2011) Pitfalls in diagnosing mitochondrial neurogastrointestinal encephalomyopathy. Journal of inherited metabolic disease 34: 11991203. 
Finkenstedt A, Schranz M, Bosch S, et al (2013) MNGIE Syndrome: Liver Cirrhosis Should Be Ruled Out Prior to Bone Marrow Transplantation. JIMD reports 10: 41-44.

Garone C, Tadesse S, Hirano M (2011) Clinical and genetic spectrum of mitochondrial neurogastrointestinal encephalomyopathy. Brain : a journal of neurology 134: 3326-3332.

Giordano C, Sebastiani M, Plazzi G, et al (2006) Mitochondrial neurogastrointestinal encephalomyopathy: evidence of mitochondrial DNA depletion in the small intestine. Gastroenterology 130: 893-901.

Gramegna LL, Pisano A, Testa C, et al (2018) Cerebral Mitochondrial Microangiopathy Leads to Leukoencephalopathy in Mitochondrial Neurogastrointestinal Encephalopathy. AJNR American journal of neuroradiology 39: 427-434.

Gronseth GS, Woodroffe LM, Getchius TSD (2011) Clinical Practice Guideline Process Manual. American Academy of Neurology

Halter JP, Michael W, Schupbach M, et al (2015) Allogeneic haematopoietic stem cell transplantation for mitochondrial neurogastrointestinal encephalomyopathy. Brain : a journal of neurology 138: 2847-2858.

Hanbali A, Rasheed W, Peedikayil MC, Boholega S, Alzahrani HA (2018) Mitochondrial Neurogastrointestinal Encephalomyopathy Syndrome Treated with Stem Cell Transplant: A Case Series and Literature Review. Experimental and clinical transplantation : official journal of the Middle East Society for Organ Transplantation 16: 773-778.

Hirano M, Marti R, Casali C, et al (2006) Allogeneic stem cell transplantation corrects biochemical derangements in MNGIE. Neurology 67: 1458-1460.

Hussein E (2013) Non-myeloablative bone marrow transplant and platelet infusion can transiently improve the clinical outcome of mitochondrial neurogastrointestinal encephalopathy: a case report. Transfusion and apheresis science : official journal of the World Apheresis Association : official journal of the European Society for Haemapheresis 49: 208-211.

Ionasescu VV, Thompson HS, Aschenbrener C, et al (1984) Late-onset oculogastrointestinal muscular dystrophy. American Journal of Medical Genetics 18: 781-788.

Kipper K, Hecht M, Antunes NJ, et al (2020) Quantification of Plasma and Urine Thymidine and 2'Deoxyuridine by LC-MS/MS for the Pharmacodynamic Evaluation of Erythrocyte Encapsulated Thymidine Phosphorylase in Patients with Mitochondrial Neurogastrointestinal Encephalomyopathy. Journal of clinical medicine 9.

Kripps K, Nakayuenyongsuk W, Shayota BJ, et al (2020) Successful liver transplantation in mitochondrial neurogastrointestinal encephalomyopathy (MNGIE). Molecular genetics and metabolism 130: 58-64.

la Marca G, Malvagia S, Casetta B, et al (2006) Pre- and post-dialysis quantitative dosage of thymidine in urine and plasma of a MNGIE patient by using HPLC-ESI-MS/MS. Journal of mass spectrometry: JMS 41: 586-592.

Lara MC, Weiss B, Illa I, et al (2006) Infusion of platelets transiently reduces nucleoside overload in MNGIE. Neurology 67: 1461-1463.

Levene M, Bain MD, Moran NF, et al (2019) Safety and Efficacy of Erythrocyte Encapsulated Thymidine Phosphorylase in Mitochondrial Neurogastrointestinal Encephalomyopathy. Journal of clinical medicine 8.

Levene M, Pacitti D, Gasson C, Hall J, Sellos-Moura M, Bax BE (2018) Validation of an Immunoassay for Anti-thymidine Phosphorylase Antibodies in Patients with MNGIE Treated with Enzyme Replacement Therapy. Molecular therapy Methods \& clinical development 11: $1-8$. 
Marti R, Spinazzola A, Tadesse S, Nishino I, Nishigaki Y, Hirano M (2004) Definitive diagnosis of mitochondrial neurogastrointestinal encephalomyopathy by biochemical assays. Clinical chemistry 50: 120-124.

Marti R, Verschuuren JJ, Buchman A, et al (2005) Late-onset MNGIE due to partial loss of thymidine phosphorylase activity. Annals of neurology 58: 649-652.

Massa R, Tessa A, Margollicci M, et al (2009) Late-onset MNGIE without peripheral neuropathy due to incomplete loss of thymidine phosphorylase activity. Neuromuscular disorders : NMD 19: 837-840.

Millar WS, Lignelli A, Hirano M (2004) MRI of Five Patients with Mitochondrial Neurogastrointestinal Encephalomyopathy. American Journal of Roentgenology 182: 15371541.

Mohamed S, Caporali L, De Giorgio R, Carelli V, Contin M (2014) HPLC-UV analysis of thymidine and deoxyuridine in plasma of patients with thymidine phosphorylase deficiency. Journal of chromatography B, Analytical technologies in the biomedical and life sciences 949-950: 5862.

Moher D, Liberati A, Tetzlaff J, Altman DG (2009) Preferred reporting items for systematic reviews and meta-analyses: the PRISMA statement. BMJ (Clinical research ed) 339: b2535.

Moran NF, Bain MD, Muqit MM, Bax BE (2008) Carrier erythrocyte entrapped thymidine phosphorylase therapy for MNGIE. Neurology 71: 686-688.

Nair R, Aggarwal R, Khanna D (2011) Methods of formal consensus in classification/diagnostic criteria and guideline development. Seminars in arthritis and rheumatism 41: 95-105.

Nishigaki Y, Martí R, Copeland WC, Hirano M (2003) Site-specific somatic mitochondrial DNA point mutations in patients with thymidine phosphorylase deficiency. The Journal of clinical investigation 111: 1913-1921.

Nishino I, Spinazzola A, Papadimitriou A, et al (2000) Mitochondrial neurogastrointestinal encephalomyopathy: An autosomal recessive disorder due to thymidine phosphorylase mutations. Annals of neurology 47: 792-800.

Oz G, Alger JR, Barker PB, et al (2014) Clinical proton MR spectroscopy in central nervous system disorders. Radiology 270: 658-679.

Pacitti D, Levene M, Garone C, Nirmalananthan N, Bax BE (2018) Mitochondrial Neurogastrointestinal Encephalomyopathy: Into the Fourth Decade, What We Have Learned So Far. Frontiers in genetics 9: 669.

Peedikayil MC, Kagevi EI, Abufarhaneh E, Alsayed MD, Alzahrani HA (2015) Mitochondrial Neurogastrointestinal Encephalomyopathy Treated with Stem Cell Transplantation: A Case Report and Review of Literature. Hematology/oncology and stem cell therapy 8: 85-90.

Roeben B, Marquetand J, Bender B, et al (2017) Hemodialysis in MNGIE transiently reduces serum and urine levels of thymidine and deoxyuridine, but not CSF levels and neurological function. Orphanet journal of rare diseases 12: 135.

Scarpelli M, Ricciardi GK, Beltramello A, et al (2013) The role of brain MRI in mitochondrial neurogastrointestinal encephalomyopathy. Neuroradiol J 26: 520-530.

Sicurelli F, Carluccio MA, Toraldo F, et al (2012) Clinical and biochemical improvement following HSCT in a patient with MNGIE: 1-year follow-up. Journal of neurology 259: 1985-1987.

Sivadasan A, Muthusamy K, Patil AK, Mathew V, Alexander M (2016) Pearls \& Oy-sters: Mitochondrial neurogastrointestinal encephalomyopathy: Diagnosis and response to peritoneal dialysis. Neurology 86: e147-e150.

Spinazzola A, Marti R, Nishino I, et al (2002) Altered thymidine metabolism due to defects of thymidine phosphorylase. The Journal of biological chemistry 277: 4128-4133. 
Torres-Torronteras J, Cabrera-Perez R, Barba I, et al (2016) Long-Term Restoration of Thymidine Phosphorylase Function and Nucleoside Homeostasis Using Hematopoietic Gene Therapy in a Murine Model of Mitochondrial Neurogastrointestinal Encephalomyopathy. Human gene therapy 27: 656-667.

Torres-Torronteras J, Cabrera-Perez R, Vila-Julia F, et al (2018) Long-Term Sustained Effect of Liver-Targeted Adeno-Associated Virus Gene Therapy for Mitochondrial Neurogastrointestinal Encephalomyopathy. Human gene therapy 29: 708-718.

Torres-Torronteras J, Viscomi C, Cabrera-Perez R, et al (2014) Gene therapy using a liver-targeted AAV vector restores nucleoside and nucleotide homeostasis in a murine model of MNGIE. Molecular therapy : the journal of the American Society of Gene Therapy 22: 901-907.

Valentino ML, Martí R, Tadesse S, et al (2007) Thymidine and deoxyuridine accumulate in tissues of patients with mitochondrial neurogastrointestinal encephalomyopathy (MNGIE). FEBS Lett 581: 3410-3414.

Yadak R, Boot MV, van Til NP, et al (2018) Transplantation, gene therapy and intestinal pathology in MNGIE patients and mice. BMC Gastroenterology 18: 149.

Yadak R, Cabrera-Pérez R, Torres-Torronteras J, et al (2018) Preclinical Efficacy and Safety Evaluation of Hematopoietic Stem Cell Gene Therapy in a Mouse Model of MNGIE. Molecular therapy Methods \& clinical development 8: 152-165.

Yavuz H, Ozel A, Christensen M, et al (2007) Treatment of mitochondrial neurogastrointestinal encephalomyopathy with dialysis. Archives of neurology 64: 435-438. 
Table 1 - Recommended brain MRI protocol.

\section{MRI technical requirements}

Brain MRI should be performed using a magnetic field of at least 1.5T with a slice thickness $=3-5 \mathrm{~mm}$ for $2 \mathrm{D}$ acquisition or $\leq 3 \mathrm{~mm}$ for $3 \mathrm{D}$ reconstruction

\section{MRI protocol}

- $\quad$ Axial 2D T2 FLAIR/T2-weighted

- $\quad$ Sagittal 2D T2-FLAIR/T2-weighted

- $\quad$ 3D T2-FLAIR/T2-weighted in alternative to axial and sagittal T2-FLAIR/T2weighted

- $\quad$ Axial DWI

- Axial T2* or SWI

- $\quad$ Axial 2D or 3D T1-weighted before and after contrast *

- $\quad$ Single voxel proton MRS sequence in white matter with signal intensity changes **

\section{Description of leucoencephalopathy (Fig. 2)}

White matter hyperintensity in T2-weighted imaging, usually bilateral, patchy and/or diffuse, periventricular and/or subcortical. It may be cloud-like in the early stage of the disease.

White matter hyperintensity generally spares U fibers, does not have mass effect or contrast enhancement and must be mostly symmetrical. Its reversibility after therapy is still under debate.

The involvement of corpus callosum, white matter capsules, basal ganglia, thalami, 
midbrain, pons, and cerebellar white matter in general has been observed in patients with long standing condition.

* MNGIE patients show no post-contrast enhancement in contrast with some white matter disorders;

** MNGIE patients show normal metabolite ratios: this is not the case for most brain white matter disorders(Oz et al 2014). Abbreviations: DWI, diffusion-weighted imaging; FLAIR, Fluid-attenuated Inversion Recovery; MRS, Magnetic Resonance Spectroscopy; SWI, Susceptibility-Weighted Imaging. 
Table 2 - Features of "Classic” and "Late Onset” phenotype of MNGIE.

\begin{tabular}{|c|c|}
\hline Classic phenotype $(n=161)^{*}$ & Late Onset phenotype $(n=8) *$ \\
\hline Age of onset $<40$ years old & Age of onset $\geq 40$ years old \\
\hline $\begin{array}{l}\text { Leukocyte TP enzymatic activity: 0-10\% } \\
\text { Plasma levels: dThd }>4 \text { and/or dUrd }>5 \\
\mu \mathrm{mol} / \mathrm{L}\end{array}$ & $\begin{array}{l}\text { Leukocyte TP enzymatic activity: 10-30\% } \\
\text { Plasma levels: dThd 0.05-4 and/or dUrd } \\
\text { 0.05-5 } \mu \mathrm{mol} / \mathrm{L}\end{array}$ \\
\hline GI symptoms $100 \%$ & GI symptoms $100 \%$ \\
\hline Leukoencephalopathy 100\% & Leukoencephalopathy 100\% \\
\hline Polyneuropathy 92-100\% & Polyneuropathy 60\% \\
\hline Ocular signs $74-100 \%$ & Ocular signs $100 \%$ \\
\hline Hearing loss $39-45 \%$ & Hearing loss $75 \%$ \\
\hline
\end{tabular}

${ }^{*}$ Number of cases described at the time of the ICC. 
Table 3 - Recommended assessments to monitor MNGIE progression.

\begin{tabular}{|c|c|c|}
\hline $\begin{array}{l}\text { Clinical/instrumental } \\
\text { assessments of disease course }\end{array}$ & Parameters & $\begin{array}{r}\text { Frequency of } \\
\text { assessment } \\
\text { (months) }\end{array}$ \\
\hline GI symptoms severity & $\begin{array}{l}\text { Abdominal pain (assessed with } \\
\text { VAS), diarrhea, vomiting, oral } \\
\text { intake (assessed with diary) }\end{array}$ & 3 \\
\hline Metabolic assessment & FGF21*, GDF15*, blood lactate & 6 \\
\hline $\begin{array}{l}\text { Body weight and body } \\
\text { composition trajectory }\end{array}$ & $\begin{array}{l}\text { BMI, prealbumin, albumin, CRP, } \\
\text { BIA (bioimpedentiometry) }\end{array}$ & 3 \\
\hline Polyneuropathy & Electroneurography & 12 \\
\hline Hepatic function and imaging & $\begin{array}{l}\text { LFTs, PT, INR, liver function } \\
\text { impairment (assessed with Child- } \\
\text { Pugh score), ultrasound, } \\
\text { elastography }\end{array}$ & $\begin{array}{l}3 \\
6\end{array}$ \\
\hline Quality of life (QoL) & SF36 & 6 \\
\hline Fatigue & FSS or FIS & 6 \\
\hline Functional status & $\begin{array}{l}\text { Karnofsky/Lansky Performance } \\
\text { Status Scale }\end{array}$ & 6 \\
\hline
\end{tabular}




\begin{tabular}{|l|l|l|}
\hline Leukoencephalopathy & $\begin{array}{l}\text { Brain MRI (no contrast agent } \\
\text { required) }\end{array}$ & \\
\hline
\end{tabular}

Abbreviations: BIA, bioelectrical impedance analysis; BMI, body mass index; CRP, C-reactive protein; FGF, fibroblast growth factor 21; FSS, fatigue severity scale; FIS, fatigue impact scale; GDF, growth differentiation factor 15; GI, gastrointestinal; INR, international normalized ratio; LFTs, liver function tests; MRI, magnetic resonance imaging; PT, prothrombin time; SF, short form 36. VAS, visual analogue scale.

* FGF21 and GDF15 are ancillary biomarkers of mitochondrial myopathy due to mtDNA maintenance defects recently established for their usefulness in documenting natural history of progression or improvements (after therapy) marking skeletal muscle in mitochondrial myopathies (Lehtonen JM, Forsström S, Bottani E, et al. FGF21 is a biomarker for mitochondrial translation and mtDNA maintenance disorders. Neurology 2016;87:2290-2299).

\section{FIGURE LEGEND}

Fig. 1 - Diagnostic algorithm in MNGIE.

Fig. 2 - Brain MRI examination from a 27-year-old severely affected MNGIE male patient. A) Axial T2-FLAIR shows bilateral and symmetrical diffuse cerebral white matter hyperintensity, with relative sparing of subcortical $U$ fibers and patchy bilateral hyperintesities in the basal ganglia, thalami and corpus callosum. B) Hyperintensities are also seen bilaterally in the pons and cerebellar white matter. (Courtesy of Prof Raffaele Lodi and Dr. Laura Ludovica Gramegna, IRCCS Istituto delle Scienze Neurologiche di Bologna). 


\section{One or more cardinal GI and neurological features referable to MNGIE including brain MRI}

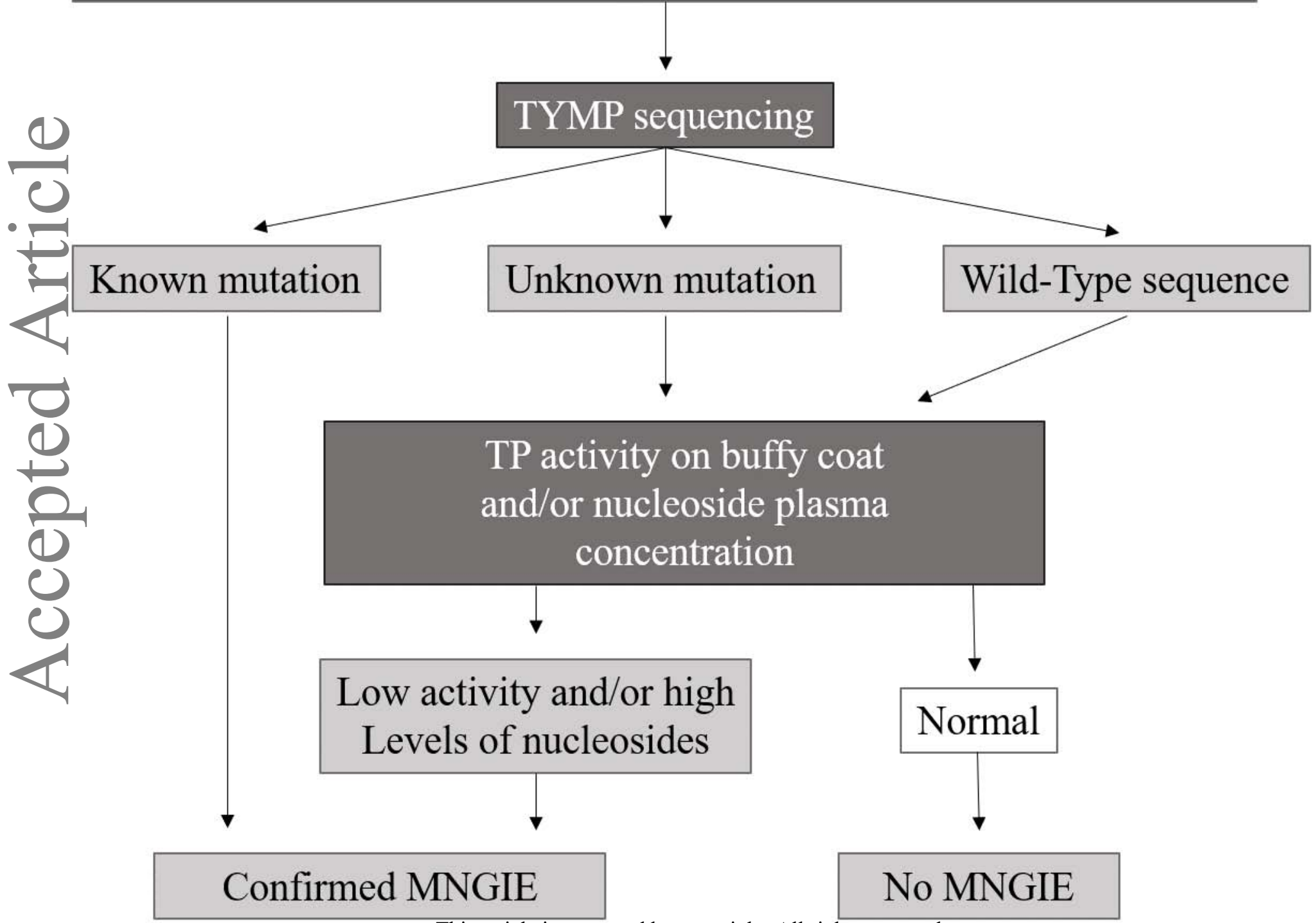

This article is protected by copyright. All rights reserved. 


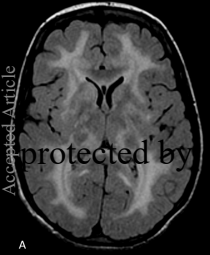

fk

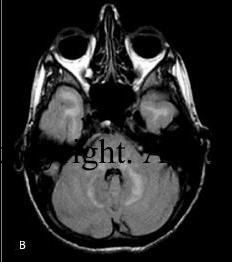

